The Prospect of using Three-Dimensional Earth Models To Improve Nuclear Explosion Monitoring and Ground Motion Hazard Assessment

T. Antoun, D. Harris, T. Lay, S. C. Myers, M. E. Pasyanos, P. Richards, A. J. Rodgers, W. R. Walter, J. J. Zucca

February 13, 2008 
This document was prepared as an account of work sponsored by an agency of the United States government. Neither the United States government nor Lawrence Livermore National Security, LLC, nor any of their employees makes any warranty, expressed or implied, or assumes any legal liability or responsibility for the accuracy, completeness, or usefulness of any information, apparatus, product, or process disclosed, or represents that its use would not infringe privately owned rights. Reference herein to any specific commercial product, process, or service by trade name, trademark, manufacturer, or otherwise does not necessarily constitute or imply its endorsement, recommendation, or favoring by the United States government or Lawrence Livermore National Security, LLC. The views and opinions of authors expressed herein do not necessarily state or reflect those of the United States government or Lawrence Livermore National Security, LLC, and shall not be used for advertising or product endorsement purposes.

This work performed under the auspices of the U.S. Department of Energy by Lawrence Livermore National Laboratory under Contract DE-AC52-07NA27344. 


\title{
The Prospect of using Three-Dimensional Earth Models To Improve Nuclear Explosion Monitoring and Ground Motion Hazard Assessment
}

\author{
Tarabay Antoun ${ }^{1}$, Dave Harris ${ }^{1}$, Thorne Lay ${ }^{2}$, Stephen C. Myers ${ }^{1}$, Michael E. Pasyanos ${ }^{1}$, \\ Paul Richards ${ }^{3}$, Arthur J. Rodgers ${ }^{1}$, William R. Walter ${ }^{1}$, John J. Zucca ${ }^{1}$ \\ ${ }^{1}$ Lawrence Livermore National Laboratory \\ ${ }^{2}$ University of California, Santa Cruz \\ ${ }^{3}$ Lamont-Doherty Earth Observatory, Columbia University
}

LLNL Technical Report LLNL-TR-401312

To be submitted to Seismological Research Letters

February 13, 2008

\begin{abstract}
Summary
The last ten years have brought rapid growth in the development and use of threedimensional (3D) seismic models of earth structure at crustal, regional and global scales. In order to explore the potential for 3D seismic models to contribute to important societal applications, Lawrence Livermore National Laboratory (LLNL) hosted a "Workshop on Multi-Resolution 3D Earth Models to Predict Key Observables in Seismic Monitoring and Related Fields" on June 6 and 7, 2007 in Berkeley, California. The workshop brought together academic, government and industry leaders in the research programs developing 3D seismic models and methods for the nuclear explosion monitoring and seismic ground motion hazard communities. The workshop was designed to assess the current state of work in 3D seismology and to discuss a path forward for determining if and how 3D earth models and techniques can be used to achieve measurable increases in our capabilities for monitoring underground nuclear explosions and characterizing seismic ground motion hazards. This paper highlights some of the presentations, issues, and discussions at the workshop and proposes a path by which to begin quantifying the potential contribution of progressively refined 3D seismic models in critical applied arenas.
\end{abstract}

\section{Introduction}

Seismic monitoring agencies are tasked with detection, location, and characterization of seismic activity in near real time. In the case of nuclear explosion monitoring or seismic hazard, decisions to further investigate a suspect event or to launch disaster relief efforts may rely heavily on real-time analysis and results. Because these are weighty decisions, monitoring agencies are regularly called upon to meticulously document and justify every aspect of their monitoring system. In order to meet this level of scrutiny and maintain operational robustness requirements, only mature technologies are considered for operational monitoring systems, and operational technology necessarily lags contemporary research. 
Current monitoring practice is to use relatively simple earth models that generally afford analytical prediction of seismic observables (see Examples of Current Monitoring Practice below). Empirical relationships or corrections to predictions are often used to account for unmodeled phenomena, such as the generation of S-waves from explosions or the effect of 3-dimensional earth structure on wave propagation. This approach produces fast and accurate predictions in areas where empirical observations are available. However, accuracy may diminish away from empirical data. Further, much of the physics is wrapped into an empirical relationship or correction, which limits the ability to fully understand the physical processes underlying the seismic observation.

Every generation of seismology researchers works toward quantitative results, with leaders who have been active at or near the forefront of what has been computationally possible. While recognizing that only a 3-dimensional model can capture the full physics of seismic wave generation and propagation in the Earth, computational seismology has, until recently, been limited to simplifying model parameterizations (e.g. 1D earth models) that lead to efficient algorithms. But what is different for us today, is that the largest and fastest machines are at last capable of evaluating the effects of generalized 3D earth structure, at levels of detail that improve significantly over past efforts, with potentially wide application. Advances in numerical methods to compute travel times and complete seismograms for 3D models are enabling new ways to interpret available data. This includes algorithms such as the Fast Marching Method (Rawlison and Sambridge, 2004) for travel time calculations and full waveform methods such as the spectral element method (SEM; Komatitsch et al., 2002, Tromp et al., 2005), higher order Galerkin methods (Kaser and Dumbser, 2006; Dumbser and Kaser, 2006) and advances in more traditional Cartesian finite difference methods (e.g. Pitarka, 1999; Nilsson et al., 2007).

The ability to compute seismic observables using a 3D model is only half of the challenge; models must be developed that accurately represent true Earth structure. Indeed, advances in seismic imaging have followed improvements in 3D computing capability (e.g. Tromp et al., 2005; Rawlinson and Urvoy, 2006). Advances in seismic imaging methods have been fueled in part by theoretical developments and the introduction of novel approaches for combining different seismological observables, both of which can increase the sensitivity of observations to earth structure. Examples of such developments are finite-frequency sensitivity kernels for body-wave tomography (e.g. Marquering et al., 1998; Montelli et al., 2004) and joint inversion of receiver functions and surface wave group velocities (e.g. Julia et al., 2000).

Improvements in data quantity and quality have also greatly increased resolution and reduced uncertainty in seismic models. The EarthScope/USArray program (http://www.earthscope.org) and similar national and international efforts are collecting large high-quality data sets that will enable 3D imaging of regional earth structure at unprecedented resolution. These efforts promise to sustain a march toward higher resolution and more accurate multi-scale 3D earth models.

To date, the primary aim of 3-dimensional model development has been to improve the understanding of earth structure and processes. Although a number of studies have 
demonstrated the utility of using 3-dimensional models for determining seismic locations (Antolik et al., 2003; Yang et al., 2004; Flanagan et al., 2007), the broader application of 3 -dimensional models in operational monitoring systems has yet to occur. Changing operational practice is a serious matter for any monitoring agency, because operational changes are costly, and there are risks to operational robustness. Further, one of the primary benefits of a longstanding operational bulletin is consistency, which enables users to place current events in the context of an established bulletin. Therefore, the only operational changes that are considered are those that will, without doubt, improve operational performance significantly.

To assess merits of incorporating 3-dimensional earth-model techniques into monitoring systems, Lawrence Livermore National Laboratory hosted a "Workshop on MultiResolution 3D Earth Models to Predict Key Observables in Seismic Monitoring and Related Fields" on June 6 and 7, 2007 in Berkeley, California (the agenda is listed in Appendix 1). This workshop brought together representatives of both the research community and operational monitoring organizations to discuss the readiness of (or prospects for) using a 3-dimensional (3D) model for seismic monitoring. In the following, we briefly summarize the current state of practice in nuclear explosion monitoring and seismic hazard analysis, then we present the current state of the art for developing 3D models and synthesizing associated 3D seismic wavefields. We conclude with a proposed path forward that was defined during the workshop, with the goals of exploiting 3D models to improve nuclear explosion monitoring and earthquake hazard assessment, and to measure progress toward these goals.

\section{Examples of Current Monitoring Practice}

The current practices for computing the many types of seismic observables are diverse and exhaustively describing them is beyond the scope of this report. Instead we provide here some examples of how simple models are currently used to predict travel times and amplitudes.

\section{Global Travel Times}

Nuclear explosion monitoring relies on one-dimensional (1D) earth models (e.g. Kennett and Engdahl, 1991) to compute a "baseline" travel-time prediction. The 1D model parameterization enables the use of analytical travel-time approaches (e.g. Buland and Chapman, 1983) that are accurate to the extent that the model is accurate. However, the $1 \mathrm{D}$ model parameterization cannot capture the physics of wave propagation in the $3 \mathrm{D}$ earth. Therefore, corrections to the baseline prediction due to any number of unmodeled effects (e.g. station elevation, earth sphericity, 3D velocity perturbations) are accounted for with travel-time corrections. In the case of corrections to account for earth ellipticity, the corrections may be model-based (Dziewonski and Gilbert, 1976). In the case of corrections to account for unmodeled velocity structure, interpolation of empirical traveltime observations may be used to improve prediction accuracy and characterize uncertainty (e.g. Figure 1; Myers and Schultz, 2000). This approach works well where empirical observations are available and has proved robust in operational systems. However, prediction accuracy diminishes away from empirical observations (both 
laterally and with event depth), and considerable effort is needed to ensure consistency of corrections for all network stations. Capturing realistic earth structure in a 3D model would not only consolidate our information about travel-times and velocities into one "container", but it would guarantee consistency of travel-time predictions for any and all seismic networks. Models can also be used to generate travel-time predictions when empirical data are sparse or unavailable, such as for a newly installed station.

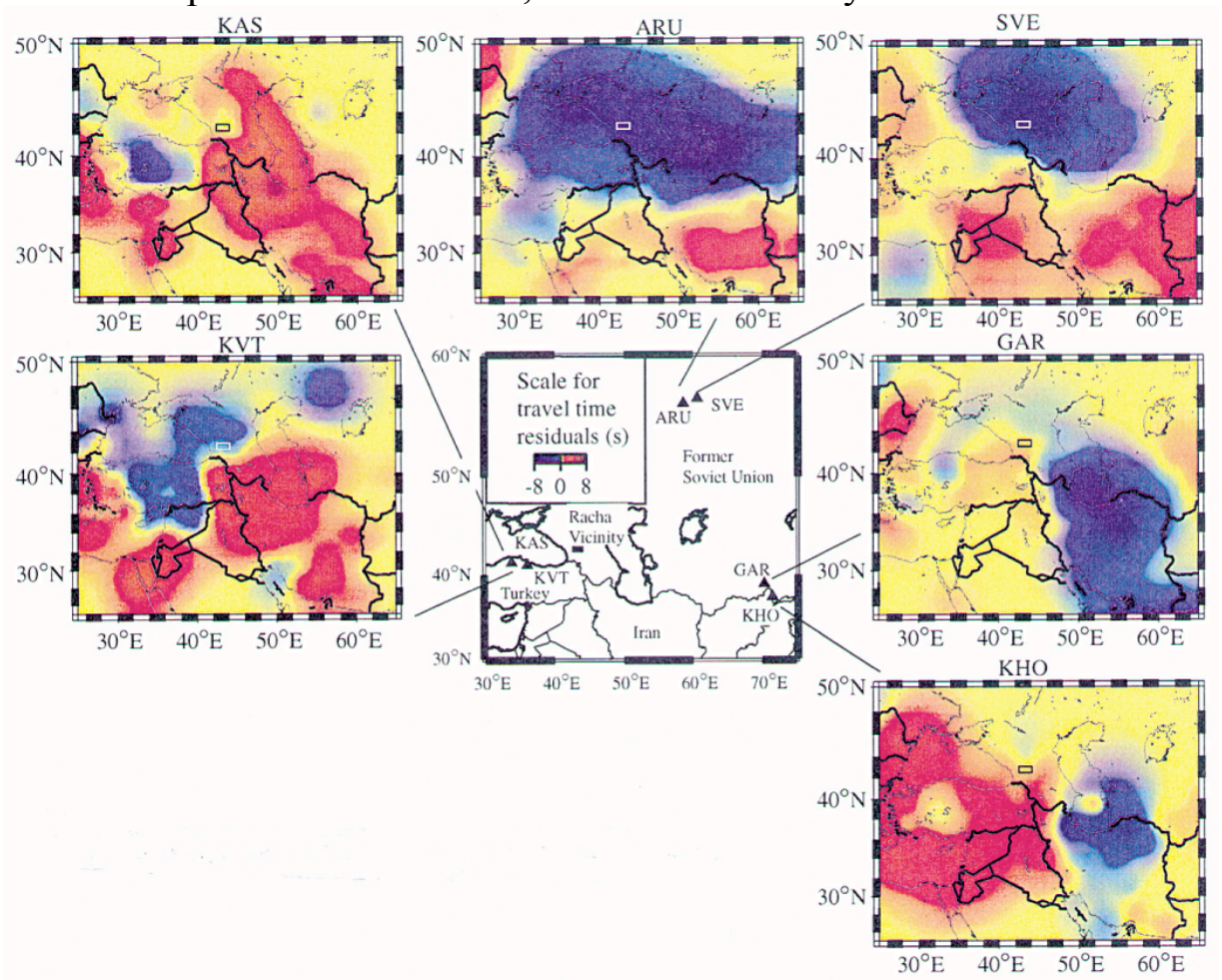

Figure 1. Empirical travel-time correction surfaces relative to a 1-D velocity model. The size of the corrections are indicated by color and range from approximately -6 to +4 seconds. Such corrections can account for tens of kilometers of location error (from Myers and Schultz, 2000).

\section{Local Amplitudes}

Ground motion calculations for seismic hazard typically rely on simple (1D) distancedependent attenuation relationships to predict ground motions (e.g. Figure 2), with corrections for site response and/or rupture directivity (e.g. Abrahamson and Silva, 1997). Site-specific corrections may be based on empirical data or generalized geotechnical corrections (e.g. NEHRP soil classifications; BSSC, 1998). However, these corrections typically do not account for path-propagation effects such as basin-generated surface waves that can have a large effect on long-period ground motions. Nor do these 1D calculations typically take into account the full time history of ground motions, such as duration of shaking, dynamic rupture, coupled structural response, and non-linear effects. 

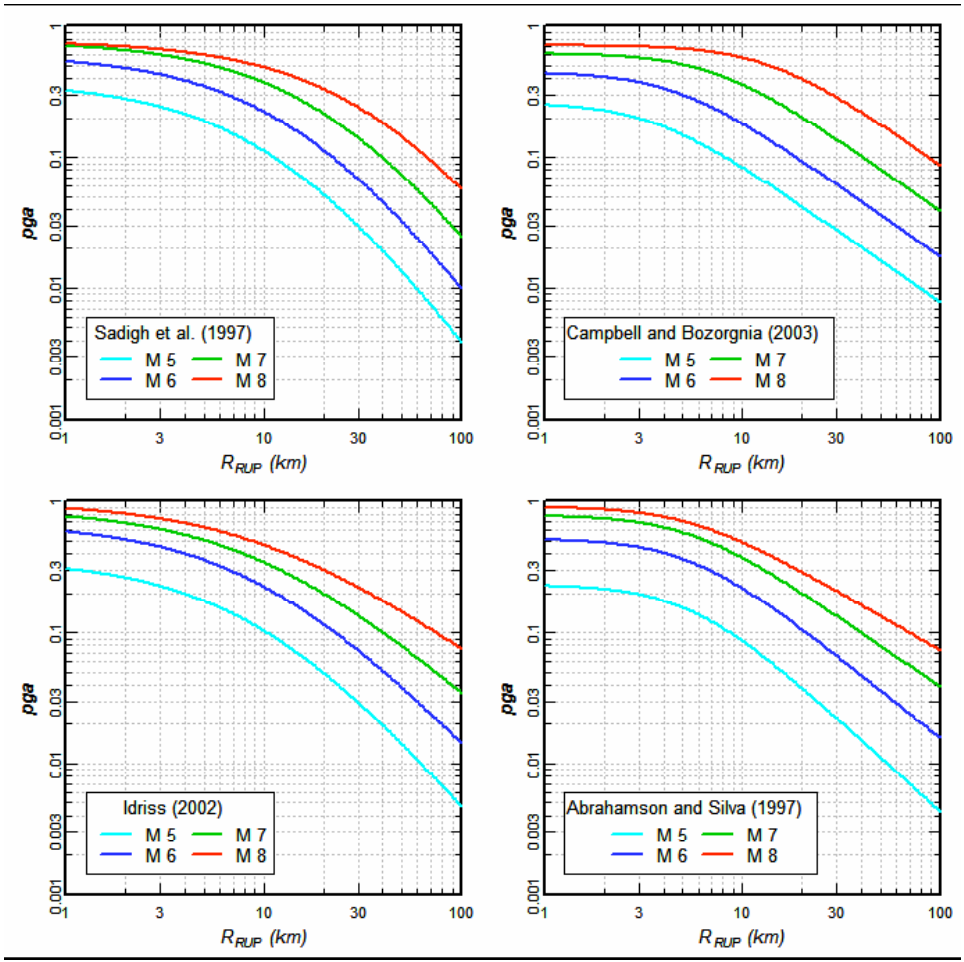

Figure 2. Peak ground acceleration (PGA) modeled as a 1D function of magnitude and distance using four attenuation formulas (Sadigh et al., 1997; Campbell and Bozorgnia, 2003; Idriss, 2002; Abrahamson and Silva, 1997). Figure from Chiou and Young, 2006.

\section{State of the Art of 3D Seismology}

Presentations at the workshop demonstrated that advances in numerical algorithms, inversion methods, computer performance and seismic data quantity and quality are enabling the development and utilization of 3D models for routine prediction of seismic parameters. First and foremost, advances in computational performance - increases in both computational power, parallel processing methods, and memory - enable prediction of seismic observables using 3D earth models with unprecedented resolution. At present, routine 3D global-scale SEM calculations are feasible with modest size computer clusters for periods as short as $\sim 10-20$ seconds (Komatitsch et al., 2002) and shorter-period calculations are possible on continental scales. Calculations for the entire globe have been performed at 3 seconds period on super computers (Komatitsch et al., 2003). Simulations of strong motions on regional scales $(\sim 500 \mathrm{~km})$ for large earthquake ruptures are possible to periods approaching $1 \mathrm{~Hz}$ (e.g. Olsen et al., 1997, 2006; Krishnan et al., 2006; Aagaard et al., 2008). Calculations of seismic attributes such as travel-time (used for location) and scalar amplitude (used for magnitude and earthquake/explosion identification) are becoming fast enough to contemplate real-time computation in a monitoring system.

While it is clear that computational methods enable forward calculations using 3D models, these calculations are only as accurate (useful) as the 3D models used to represent earth structure. At the workshop there was a general concern from nuclear 
explosion and hazard organizations whether model resolution is or will become adequate to significantly improve prediction accuracy of seismic observables at relevant frequencies.

Several workshop speakers focused on model construction and inverse methods to improve model resolution. At local distances (event-station separation of $<\sim 100 \mathrm{~km}$ ), geologic mapping and the use of seismic exploration methods (e.g., seismic reflection/refraction and potential field analysis) have been used to resolve 3D structure of the upper crust and successfully improve prediction of seismic ground motion (presentations by Brocher and Bielak). There have been successful 3D model-building efforts in the seismic hazard community. The Southern California Earthquake Center (SCEC) Community Modeling Environment (SCEC, 2007) provides information technology infrastructure for seismic hazard analysis (Jordan et al., 2003). This effort is both flexible and extensible and has resulted in state of the art ground motion forward (Olsen et al., 2006) and inverse (Akcelik et al., 2003) calculations. The recently developed USGS 3D geologic and seismic velocity model of the San Francisco Bay Area (Figure 3) successfully reproduces ground motions from moderate regional earthquakes (Rodgers et al., 2008) and the intensity data from the 1906 San Francisco earthquake (Aagaard et al., 2008). Furthermore, the open and collaborative nature of community modeling has given end users transparency into model construction.

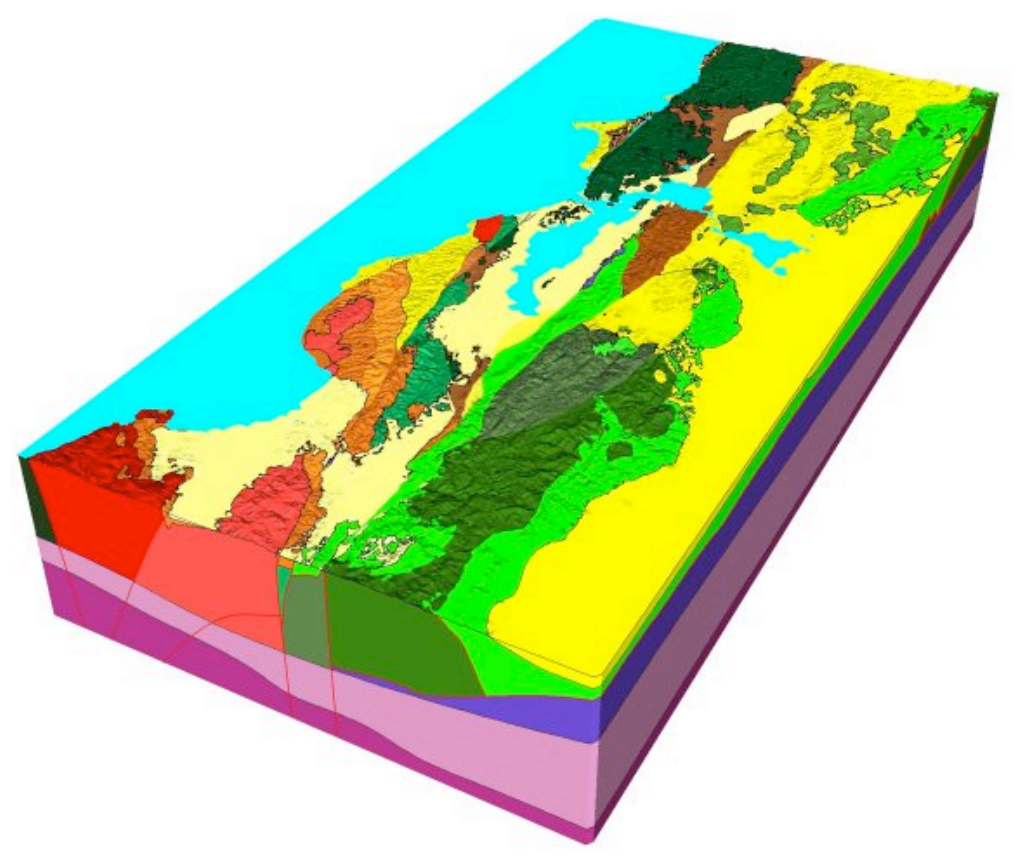

Figure 3. USGS 3D geologic and seismic model of the San Francisco Bay Area. Colors denote distinct geological units. Figure from Brocher, 2005.

While innovative, the data used in the construction of the Bay Area model and SCEC Community Model (i.e. high concentration of wells and well logs, very dense station spacing) are generally not available over the broader globe. At regional distances $(\sim 1000$ 
$\mathrm{km})$ surface- and body-wave techniques can robustly resolve 3D aspects of crustal and upper-mantle structure (presentations by Pasyanos, Ammon, and Thurber). Even so, broad areas of the globe (aseismic regions; oceanic regions and other areas that for political or logistical reasons do not have seismic stations) will be sampled only with limited body waves paths such as multiple surface reflections (PP, PPP, SS, etc.) and surface waves, which generally have lower resolution and may not be sensitive enough to discontinuities important for travel time and amplitude prediction. Accordingly, several talks in the session focused on the advantages of developing seismic models using multiple constraints, including the gravity field (presentations by Pasyanos and Ammon). Discussion highlighted the importance of the starting model in these inversions, as well as ways of estimating model uncertainty. Probabilistic methods such as Markov Chain Monte Carlo (MCMC) and Neighborhood Algorithm (NA) were discussed as possible estimators of uncertainty.

One recent advance is the use of seismic noise to extract inter-station Green functions and, combined with traditional surface wave dispersion measurements, to determine 3D structure from the surface to several hundred kilometers depth, thus enabling model development in the absence of discrete seismic sources (presentation by Ritzwoller). At the global scale, free oscillations of the Earth and body-wave techniques are proven methods for improving Earth model accuracy (presentation by Shearer). An exciting recent development is the application of adjoint methods to use the complete waveforms and finite-frequency sensitivities to update 3D structure (e.g. Figure 4), using computationally intensive forward and back-projection calculations (presentation by Tromp). Seismic tomography based on adjoint methods can be used to improve models at local, regional and global scales, and holds the promise of resolving the $3 \mathrm{D}$ structure needed for full waveform prediction.

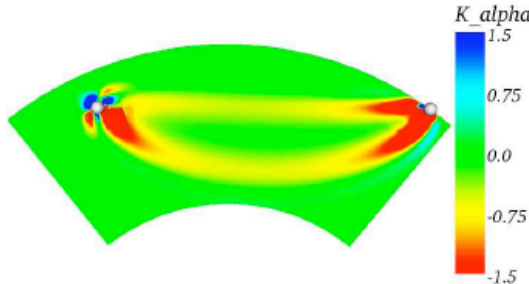

(a)

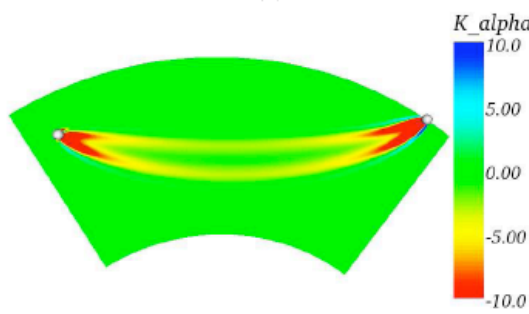

(c)

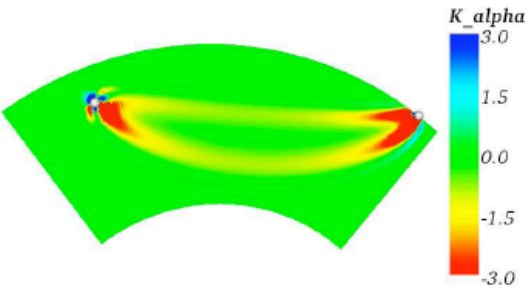

(b)

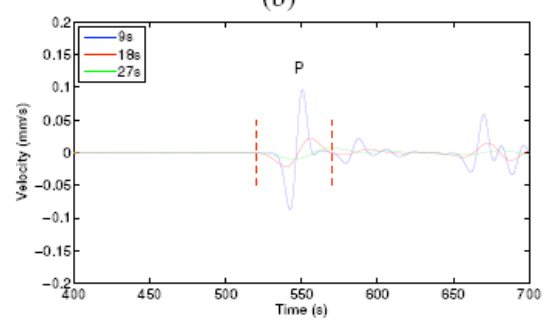

(d)

Figure 4. An example of an SEM method to compute adjoint kernels for estimating 3D structure. Red (-) and blue (+) show areas of sensitivity. Figure from Liu and Tromp, 2006. 


\section{Discussion}

The workshop included an open-floor discussion that we summarize here. Provided that model predictions are properly validated with appropriate empirical data, employing 3D earth models has the potential to make major improvements in applying seismic technology to important societal issues. In nuclear explosion monitoring, for example, there is the possibility in the near term to use 3D earth models based on simple tectonic regionalization (e.g. Pasyanos et al., 2004; Johnson and Vincent, 2002) to develop travel time correction surfaces in regions where empirical ground-truth events are lacking such as in North Africa and large regions of the former Soviet Union (e.g. Flanagan et al., 2007). The use of 3D models has the additional advantage of reducing the nonstationarity of empirical corrections that are made relative to these models. If they are validated, 3D models can be developed to replace the current regionalized 1D models, making possible self-consistent calculations of travel times and waveforms from local to regional to teleseismic distances.

For studies of seismic hazard, 3D models can be used to improve event locations for defining seismicity and more importantly for first-principles calculation of ground motion for scenario earthquakes. This will allow modeling of ground motion hazard for a large number of scenarios including variations in rupture directivity and path-propagation effects. Large $\left(\mathrm{M}_{\mathrm{W}}>6.0\right)$ damaging earthquakes are infrequent and near-fault observations of ground motion time-series are limited to sparse numbers of recording stations. Consequently the database of recorded ground motions grossly undersamples the population of possible motions and near-fault locations. Simulations of ground motions in 3D models make it possible to compute estimates of the earth's response anywhere for a wide range of potentially damaging earthquakes. The simulated ground motion time-series can feed directly into building response calculations for performancebased design of structures (Krishnan et al., 2006).

While the potential to improve monitoring using 3D models was not in question, monitoring agencies questioned the readiness of the technology for real-time applications. There are many well-known concerns/conditions that a 3D model approach would have to address (e.g. Harris et al., 2007). Some of these were discussed at the meeting, particularly issues related to how best to parameterize models and exchange them between research groups (presentation by Menke). In the next section we consolidate and summarize the major issues.

\section{Considerations on the Use of 3D Models}

Model building using measurements derived from geophysical observables is conceptually simple as illustrated in Figure 5. 


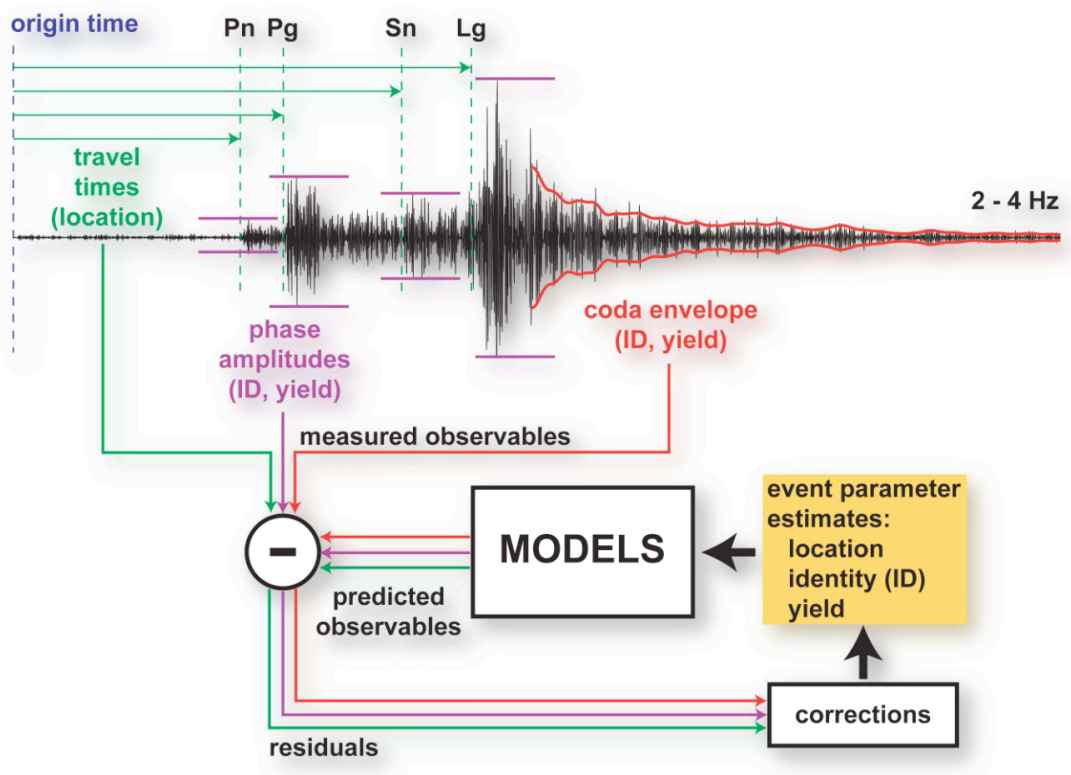

Figure 5. Models form the core of an iterative process of predicting observed signal characteristics (parameters, envelopes, waveforms) in order to detect, locate, identify, and characterize seismic events (from Harris et al., 2007).

In practice many questions arise when contemplating a transition from 1-D, 2-D and 2.5D models (restrictively parameterized 3D) to constructing and using 3-D models (e.g. Harris et al., 2007; Menke presentation at conference and conference discussion), among them:

1) Can a 3-D geophysical model or a collection of 3-D models provide measurably improved predictions of seismic monitoring observables over existing or planned 1-D models, or $2-\mathrm{D}$ and $2 \frac{1}{2}-\mathrm{D}$ models?

2) Is a single model that can predict all observables achievable, or must separate models be devised for each observable? How should joint inversion of disparate observable data be performed, if required?

3) What are the options for model representation? Can common and well-defined parameterizations be set to encourage quantitative exchanges of models between researchers? How does representation affect the accuracy and speed of observable predictions? Are multi-resolution models essential?

4) How should model uncertainty be estimated, represented, and how should it be used? Are stochastic models desirable? How are the models and their uncertainties to be validated in non-circular ways?

5) What data types should be used to construct the models? What quality control regime should be established? How do we best encourage researchers to make their underlying data available? 
6) How will 3-D models be used in operational practice? Will significant improvements in the basic functions (e.g. detection, location, characterization) result from the use of 3D models? Will the calculation of observables through 3-D models be fast enough for real-time use or must a strategy of pre-computation, which limits the flexibility of the network, be employed?

7) What are the theoretical limits to 3-D model development (resolution, uncertainty) and performance in predicting monitoring observables? How closely can those limits be approached with projected data availability, station distribution and inverse methods?

8) What priorities should be placed on the acquisition of event ground truth information, deployment of new stations, development of new inverse techniques, exploitation of large-scale computing, and other activities in the pursuit of 3-D model development and use?

These questions have many possible answers and provoked much of the discussion at the workshop. They leave open a very wide-ranging field of future research in 3D geophysical models. In order to reach the most practically useful results in the shortest possible time there needs to be a way to move towards community consensus among the possible paths forward, such as occurred in Southern California with the SCEC Community Modeling Environment, or in Northern California with the USGS 3D Bay Area Model. The last part of the workshop addressed such specific proposals.

\section{Possible Paths Forward}

There was a consensus that demonstration of prediction accuracy and robustness using 3D models was needed. Two specific proposals were considered and found to have merit during the Path Forward discussion at the workshop. The first was for the development of an Earth Model Framework Test Bed by the nuclear explosion monitoring community. A stated goal of such a model is to be relevant across all distance scales. Local and regional models are generally developed making simplistic assumptions about the deep earth (i.e., they commonly connect smoothly onto a 1D model like iasp91). Similarly, 3D models of the mantle make similar simplistic assumptions about the crust, usually employing a crustal model like CRUST5.1 (Mooney et al., 1998) or CRUST2.0 (Bassin et al., 2000). The result is an inconsistent model across the distance scale. There is a strong need to reconcile crustal/upper-mantle models and lower-mantle models, so that the resulting model consistently explains regional and teleseismic data.

The Earth Model Framework Test Bed would involve the construction two models linked through a common crustal, lithospheric and upper mantle discontinuity structure. The models would be intended to predict teleseismic and regional $\mathrm{P}$ travel times in order to improve event location estimates (the 'P model') and to predict surface-wave dispersion measurements and to estimate $\mathrm{M}_{\mathrm{S}}$ (the 'S model'). The model could be combined with density and attenuation for full waveform calculations, if necessary. The models were proposed to be both hierarchical and multi-resolution to provide increased resolution 
where justified by data coverage. A common format and shared structure would allow the models to be merged into a single model at some point in the future. The overriding consideration in development of such a model pair was to pick a practical goal as a starting point for a collaborative effort with sufficient flexibility to be extendable to more complex models, if warranted. There also were strong requirements to develop models immediately useful for monitoring objectives and perform non-circular validation. For example, a realistic goal of locating earthquakes would be to universally reduce the $2 \sigma$ epicenter errors from $20 \mathrm{~km}$ to $5 \mathrm{~km}$ for a data set spanning regional and teleseismic distances, at least for events with $\mathrm{M} \geq 5.0$. A similar metric could be made for event identification. If the models perform adequately they could be combined and serve as a starting model for advanced imaging methods, such as joint inversion of multiple data types or seismic waveform tomography with adjoint methods.

An idealized goal is to have a single global 3D elliptical Earth model with Vp, Vs, $\rho$, Qp, Qs, and anisotropy parameters (along with corresponding uncertainty values) that is able to predict the spectrum of geophysical and seismological observations. The more modest current proposal is to develop a model that, while limited in scope, improves performance over current methods, works efficiently in an operational environment, and is flexible and expandable to accommodate future development (Figure 6). The near term goal of such a model would be the ability to significantly improve the fit of derived seismic observables (e.g. travel times, amplitudes) and predicting waveforms at long periods. The long-term goal would be to improve the resolution and sophistication of such models (anisotropy, etc.) to predict waveforms at increasingly higher frequency.

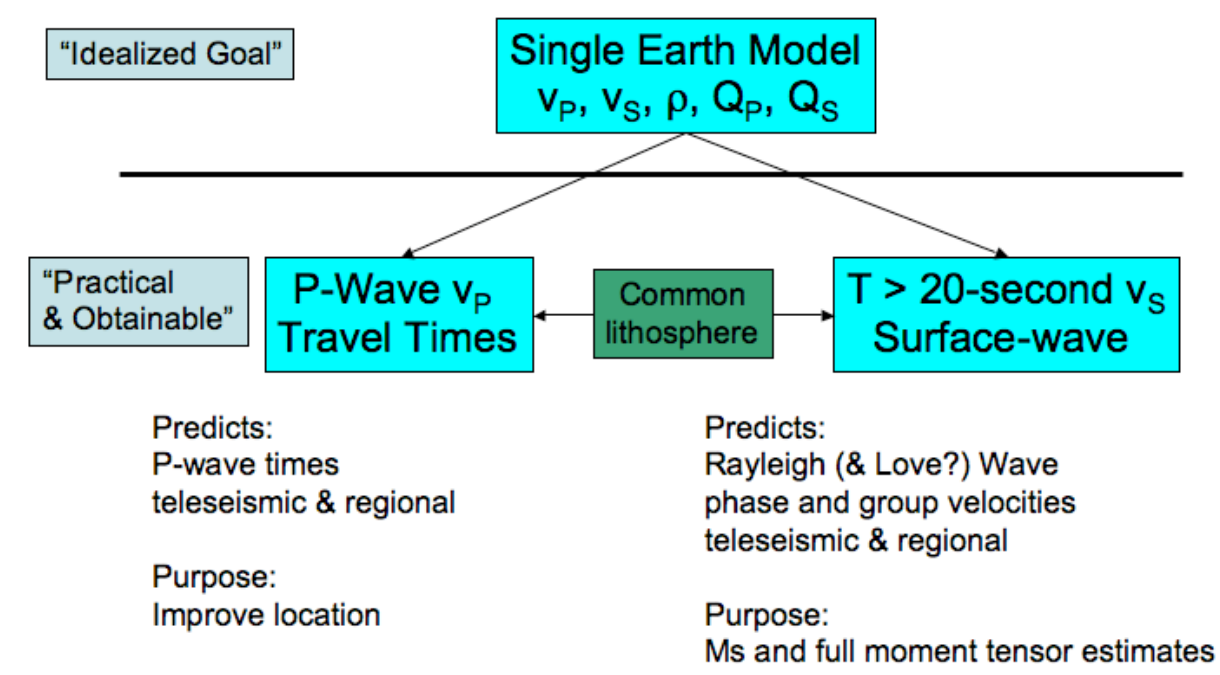

Figure 6. Proposal for the Earth Model Framework Test Bed.

A second proposed path forward was designed to specifically address one of the most critical challenges for explosion seismology, that is, to explain the frequency dependent behavior of the S-waves generated by explosions. It is widely observed that the ability to identify small explosions depends on the relative amplitude of regional $\mathrm{P} / \mathrm{S}$ ratios above about $3 \mathrm{~Hz}$. Existing 3D elastic wave propagation algorithms running on high performance computing could be used to address this question, however it is nearly 
impossible to obtain the correct characterization of the subsurface structure at the resolution required for such calculations. While full waveform calculations at regional distances may be challenging, it may be possible to evaluate the relative contributions of various factors by including more complete descriptions of the physics of explosions. These include rock fracture, spall, heterogeneity and topographic scattering, tectonic stress release and other effects. The objective of such an effort would be to create a physical model that is ultimately able to make predictions in new regions and under testing conditions for which no empirical data exists.

To accomplish this goal, sophisticated 3D models of the source region would need to be developed. If models with sufficient resolutions are available, existing, well-developed modeling codes for non-linear near source effects (Figure 7), such as GEODYN (Lomov and Rubin, 2003; Antoun et al., 2006) are available to compute the non-linear effects of the explosion source out to the elastic radius. These could be leveraged to tie to existing local scale seismic observations if three critical issues can be overcome. First motions from the non-linear source region codes need to be passed across domain boundaries to elastic (linear) finite difference or spectral element method codes and propagated to regional distances. Second, the large differences in spatial and temporal scales between the two methodologies needs to be handled. Third, 3D source region models around past nuclear tests with good empirical data need to be built, used in 3D calculations and validated (matching available pressure and ground motion data) to provide confidence in the results.

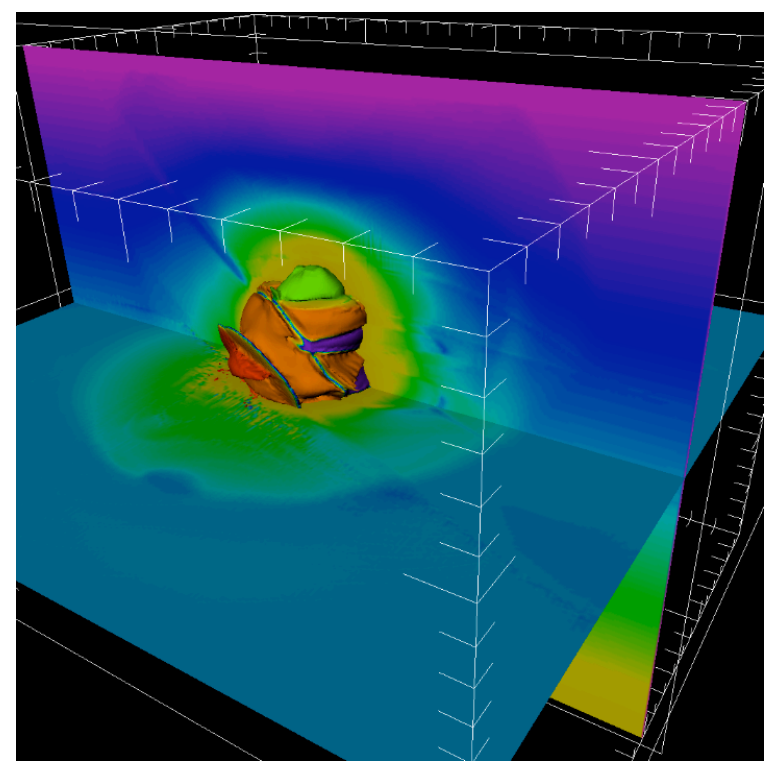

Figure 7. A composite image from a three-dimensional GEODYN simulation showing pressure contours superimposed on complex geologic features that characterized the cavity region of the BANEBERRY underground nuclear test. Different colors on the pressure isosurface indicate different materials, and the two diagonally slanting lines are faults that cut through the geology near the cavity. The image underscores the importance of material properties, heterogeneities and geologic structure on non-linear wave propagation through geologic media (Lomov et al. 2003). 
The ability to tie non-linear source region modeling to seismic finite difference codes would allow many additional investigations of interest both to the seismic monitoring community and the earthquake hazard community. For example a single set of codes that covered calculations from non-linear source region to the linear region where most seismic records are made allows a number of scenarios: 1) investigations of non-linear source effects in earthquakes (e.g. super-shear rupture; rupture induced fracture, triggering of rupture); 2) non-linear source-structure interactions of critical importance to earthquake hazard analysis (e.g. non-linear soil response, liquefactions, soil-structure interactions); and 3) ties to existing seismic records for model development and validation.

\section{Conclusion}

As a result of recent advances in theory, data, and computational power, there is a new prospect for developing and using 3D models for monitoring applications. Conditional to these developments, however, are the expectations that 1) the models will be built in a transparent manner using a variety of seismological and geophysical observations; 2) the $3 \mathrm{D}$ models represent a significant improvement over current methods (1D, 1D with corrections, and 2D, using community consensus metrics); and 3) implementation of the models will not be restrictively slow and difficult to use for realistic operations.

Furthermore, these models would have to be cognizant of the considerations on the use of 3D models.

Two specific proposals were advanced during the workshop. The first is for development of a P- and S-wave model of the crust and mantle (to the core), as well as methods for computing self-consistent travel-times at local, regional, and teleseismic distances. This proposal is specifically limited in scope, resolution, and complexity and is meant to demonstrate capability in the monitoring arena in the near-term. The second proposal was for a model that would explain the frequency dependent behavior of S-waves generated by explosions. This would be accomplished by coupling codes for non-linear source region modeling to seismic finite difference codes. Because they each try to address separate specific questions, they are not mutually exclusive proposals and could be advanced simultaneously if resources allow.

\section{Acknowledgements}

We are grateful for the workshop participants for attending and, especially, the speakers for taking the time and energy to prepare their excellent presentations. We also thank David McCallen for support and encouragement for the workshop. This would not have been a success without logistical support from Connie Ruvalcaba-Olson and Sandi Gonsalves. This work performed under the auspices of the U.S. Department of Energy by Lawrence Livermore National Laboratory under Contract DE-AC52-07NA27344. This is LLNL contribution LLNL-TR-401312. 


\section{References}

Aagaard, B., T. Brocher, D. Dolenc, D. Dreger, R. Graves, S. Harmsen, S. Hartzell, S. Larsen, K. McCandless, S. Nilsson, A. Petersson, A. Rodgers, B. Sjogreen and M. L. Zoback (2008). Ground motion modeling of the 1906 San Francisco earthquake II: Ground motion estimates for the 1906 earthquake and scenario events, in press Bull. Seism. Soc. Amer.

Abrahamson, N.A., and W.J. Silva (1997), Empirical response spectral attenuation relations for shallow crustal earthquakes, Seism. Res. Lett., 68, 94-127.

Akcelik, V., J. Bielak, G. Biros, I. Ipanomeritakis, A. Fernandez, O. Ghattas, E. Kim, D. O'Hallaron, and T. Tu (2003), High Resolution Forward and Inverse Earthquake Modeling on Terasacale Computers, SumperComputing 2003, Phoenix, AZ.

Antolik, M., Y.J. Gu, G. Ekstrom and A.M. Dziewonski (2003). J362D28: a new joint model of compressional and shear velocity in the Earth's mantle, Geophys. J. Int, 153, (2), 443-466.

Antoun, T. H., L.A. Glenn, O.R. Walton, P. Goldstein, I.N. Lomov, and B. Liu (2006), Simulation of hypervelocity penetration in limestone, Int. J. Impact Eng., 33, 4552.

Bassin, C., G. Laske, and G. Masters (2000), The current limits of resolution for surface wave tomography in North America, EOS Trans AGU, 81, F897.

Brocher, T.M. (2005), Compressional and shear wave velocity versus depth in the San Francisco Bay Area, California: Rules for USGS Bay Area Velocity Model 05.0.0, USGS Open-File Report 05-1317.

Building Seismic Safety Council (BSSC) (1998). National Earthquake Hazard Reduction Program recommended provisions for seismic regulations for new buildings and other structures, 1997 Edition, FEMA 302/303, developed for the Federal Emergency Management Agency, Washington, DC.

Buland, R. and C.H. Chapman (1983). The computation of seismic travel times, Bull. Seismol. Soc. Am., 73, 1271-1302.

Campbell, K.W., and Y. Bozorgnia (2003), Updated near-source ground-motion (attenuation) relations for the horizontal and vertical components of peak ground acceleration and acceleration response spectra, Bull. Seism. Soc. Amer., 93, 314331.

Dumbser, M. and M. Käser (2006). An arbitrary high-order discontinuous Galerkin method for elastic waves on unstructured meshes - II. The three-dimensional isotropic case, Geophys. J. Int., 167, 319-336.

Dziewonski, A.M., and F. Gilbert (1976). The effect of small, aspherical perturbations on travel times and a re-examination of the corrections for ellipticity, Geophys. J. R. astr. Soc., 44, 7-17.

Flanagan, M., S. Myers and K. Koper (2007). Regional Travel-Time Uncertainty and Seismic Location Improvement Using a Three-Dimensional a priori Velocity Model, Bull. Seismol. Soc. Am. 97, 804 - 825.

Harris, D.B., J.J. Zucca, D. McCallen, M.E. Pasyanos, M.P. Flanagan, S.C. Myers, W.R. Walter, A.J. Rodgers, and P.E. Harben (2007), Considerations on the use of 3-D geophysical models to predict test ban monitoring observables, 29th Monitoring 
Research Review: Ground-Based Nuclear Explosion Monitoring Technologies, Denver, Colorado, September 25-27, 2007.

Idriss, I.M., (2002) Personal Communication.

Johnson, M., and C. Vincent (2002). Development and testing of a 3D velocity model for improved event location: A case study for the India-Pakistan region, Bull. Seism. Soc. Am. 92, 2893-2910.

Jordan, T., P. Maechling, and SCEC/CME Collaboration (2003). The SCEC Community Modeling Environment - An Information Infrastructure for System-Level Earthquake Science, Seism. Res. Lett. 74, 3, 44-46.

Joyner, W.B., and D.M. Boore (1981), Peak horizontal acceleration and velocity from strong-motion records including the records from the 1979 Imperial Valley, California, earthquake, Bull. Seism. Soc. Amer., 71, 2011-2038.

Julia, J., C.J. Ammon, R.B. Herrmann, and A.M. Correig (2000). Joint inversion of receiver function and surface wave dispersion observations, Geophys. J. Int., 143, $1-19$.

Käser, M. and M. Dumbser (2006). An arbitrary high-order discontinuous Galerkin method for elastic waves on unstructured meshes - I. The two-dimensional isotropic case with external source terms, Geophys. J. Int., 166, 855-877.

Kennett, B.L.N. and E.R. Engdahl (1991). Traveltimes for global earthquake reference location and phase identification, Geophys. J. Int, 105, 429-465.

Komatitsch, D., J. Ritsema and J. Tromp (2002). The Spectral-Element Method, Beowolf computing and global seismology, Science, 298, 1737-1742.

Komatitsch, D., S. Tsuboi, J. Chen, and J. Tromp (2003). A 14.6 billion degrees of freedom, 5 teraflops, 2.5 terabyte earthquake simulation on the Earth Simulator, Proceedings of the Supercomputing 2003 Conference.

Krishnan, S., J. Chen, D. Komatitsch, and J. Tromp (2006). Performance of 18-story steel moment-frame buildings in Southern California during a large San Andreas earthquake, Earthquake Spectra, 22, 1035-1061.

Liu, Q., and J. Tromp (2006). Finite-frequency kernels based upon adjoint methods, Bull. Seism. Soc. Amer., 96, 2383-2397.

Lomov, I., T. Antoun, J. Wagoner, and J. Rambo (2003), Three Dimensional Simulation of the Baneberry Nuclear Event, 13th APS Topical Conference on Shock Compression of Condensed Matter, Portland, OR, July 20-25 2003.

Lomov, I. and M.B. Rubin (2003), Numerical simulation of damage using an elasticviscoplastic model with directional tensile failure, J. Phys. IV, 110, 281-286.

Marquering, H., G. Nolet and F.A. Dahlen (1998). Three-dimensional waveform sensitivity kernels, Geophys. J. Int., 132, 521-534.

Montelli, R., G. Nolet, F.A. Dahlen, G. Masters, E.R. Engdahl, and S.-H. Hung (2004), Finite frequency tomography reveals a variety of plumes in the mantle, Science, 303, 338-343.

Mooney, W.D., G. Laske, and T.G. Masters (1998), CRUST 5.1: A global crustal model at $5^{\circ} \times 5^{\circ}$, J. Geophys. Res., 103, 727-747.

Myers, S.C., and C.A. Schultz (2000). Improving sparse network seismic location with Bayesian kriging and teleseismically constrained calibration events, Bull. Seismol. Soc. Am., 90, 199-211. 
Nilsson, S., N. A. Petersson, B. Sjogreen, and H.-O. Kreiss (2007). Stable difference approximations for the elastic wave equation in second order formulation, SIAM J. Numer. Anal., 45, 5, 1902-1936, doi:10.1137/060663520.

Olsen, K. B., R. Madariaga, and R. J. Archuleta (1997). Three-dimensional dynamic simulation of the 1992 Landers earthquake, Science, 278, 834-838.

Olsen, K. B., S. M. Day, J. B. Minster, Y. Cui, A. Chourasia, M. Faerman, R. Moore, P. Maechling, and T. Jordan (2006), Strong shaking in Los Angeles expected from southern San Andreas earthquake, Geophys. Res. Lett., 33, L07305.

Pasyanos, M.E. (2000), Predicting geophysical measurements: testing a combined empirical and model-based approach using surface waves, Bull. Seismo. Soc. Am., 90, 3, 790-796.

Pasyanos, M.E., W.R. Walter, M.P. Flanagan, P. Goldstein, and J. Bhattacharyya (2004), Building and testing an a priori geophysical model for Western Eurasia and North Africa, Pure Appl. Geophys., 161, 235-281.

Pasyanos, M.E., G.A. Franz, and A.L. Ramirez (2006), Reconciling data using Markov Chain Monte Carlo: An application to the Yellow Sea - Korean Peninsula region, J. Geophys. Res., 111, B03313, doi:10.1029/2005JB003851.

Pitarka, A. (1999). 3D Elastic finite-difference modeling of seismic motion using staggered grids with nonuniform spacing, Bull. Seismo. Soc. Am., 89, 54-68.

Rawlinson, N. and M. Sambridge (2004). Wavefront evolution in strongly heterogeneous layered media using the fast marching method, Geophys. J. Int., 156, 631-647.

Rawlinson, N. and M. Urvoy (2006). Simultaneous inversion of active and passive source datasets for 3-D seismic structure with application to Tasmania, Geophys. Res. Lett., 33, L24313.

Rodgers, A., A. Petersson, S. Nilsson, B. Sjogreen and K. McCandless (2008). Broadband waveform modeling of moderate earthquakes in the San Francisco Bay Area and preliminary assessment of the USGS 3D seismic velocity model, in press Bull. Seism. Soc. Amer.

Sadigh, K., C.-Y. Chang, J.A. Egan, F.I. Makdisi, and R.R. Youngs (1997), Attenuation relationships for shallow crustal earthquakes based on California strong motion data, Seism. Res. Lett., 68, 1, 180-189.

Sambridge, M. (1999). Geophysical inversion with a neighbourhood algorithm - I. Searching a parameter space, Geophys. J. Int., 138, 479-494.

SCEC Community Modeling Environment (2007), http://epicenter.usc.edu/cmeportal/index.html.

Tromp, J., C. Tape, and Q. Liu (2005). Seismic tomography, adjoint methods, time reversal, and banana-donut kernels, Geophys. J. Int., 160, 195-216.

Yang, X.P., I. Bondar, J. Bhattacharyya, et al. (2004). Validation of regional and teleseismic travel-time models by relocating ground-truth events, Bull. Seism. Soc. Am., 94, 897-919. 
Appendix 1 (remove for article submission)

\title{
Dav 1 - Wednesdav, June 6,2007
}

7:45-8:15 Arrive and Register (Angel Foyer / Angel Room)

8:15-8:25 Greetings and opening remarks (Continental Breakfast provided)

Jay Zucca, LLNL

8:25-8:40 Comments on the use of 3-dimensional models for monitoring David Russell, AFTAC

8:40-9:00 "Key observables for monitoring hazard: the prospect of using 3D models to improve prediction"

\section{David McCallen, LLNL}

\author{
SESSION I: Model Building Using Geologic Compilation Approaches and \\ Considerations of Model Parameterization \\ 9:00-9:40 "Building a better SF Bay Area model" \\ Thomas Brocher, USGS \\ 9:40-10:20 "The evolution of global and regional models" \\ Michael Pasyanos, LLNL \\ 10:20-10:50 Break/Informal Discussions (Refreshments) \\ 10:50-11:30 "Representation of Global Earth Models: efficiency, interoperability \\ and prediction accuracy" \\ William Menke, Lamont-Doherty Earth Observatory \\ 11:30-12:00 Discussions \\ 12:00-1:00 Working Lunch Provided (Discussions) (Bay Grille Restaurant)

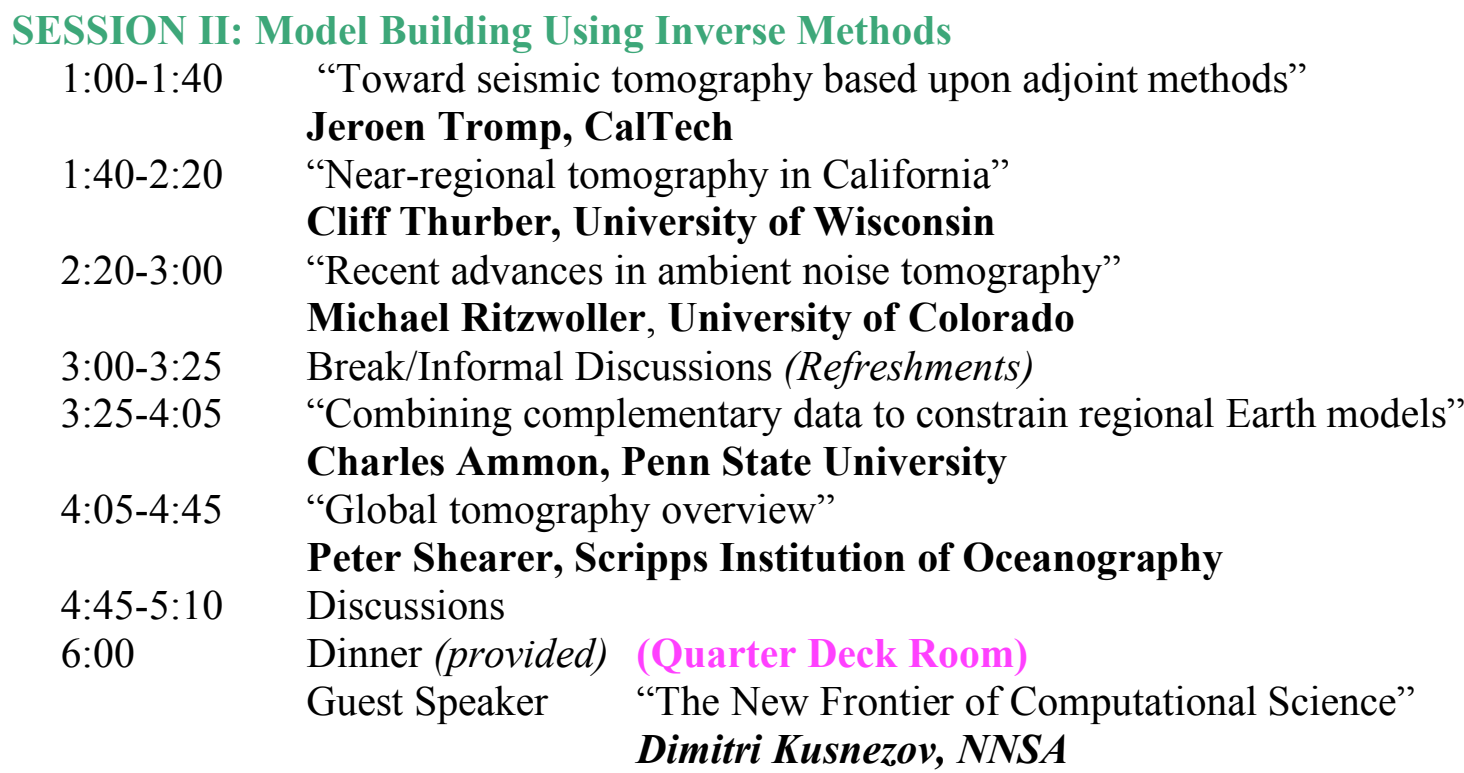


Dav 2 - Thursdav, June 7, 2007

\author{
7:45-8:00 Arrive (Angel Room) \\ 8:00-8:15 Greetings and recap of previous day Jay Zucca, LLNL (Continental \\ Breakfast provided)
}

SESSION III: Seismic Observables in Monitoring and Earthquake Hazard (Current accuracy of prediction)

8:15-8:55 "Three-dimensional simulation in seismic hazard assessment for engineering practice"

8:55-9:35 "End-to-end earthquake modeling: From 3D ground motion to structural performance, including soil-structure interaction" Jacobo Bielak, Carnegie Mellon University

9:35-10:00 Break/Informal Discussions (Refreshments)

10:00-10:40 "Non-linear source modeling and coupling to linear codes" Tarabay Antoun, LLNL

10:40-11:20 "Modeling regional broadband arrays" Donald Helmberger, Cal. Inst. Tech.

11:20-12:00 Discussions

12:00-1:00 Working Lunch Provided (Discussions) (Bay Grille Restaurant)

SESSION IV: Technology Path Forward

1:00-2:45 Thorne Lay, UCSC and David McCallen, lead a discussion on forward calculations. Given computational methods and resources (hardware) projected in the next 10 years, to what frequencies and distances might we compute synthetic seismograms from 3D models? (La Jolla Room)

2:45-3:15 Break/Informal Discussions (Refreshments)

3:15-5:00 Paul Richards, Columbia and Jay Zucca, lead a discussion on model resolution. Given inverse (tomographic) methods and data sets anticipated to be available over the next 10 years, what model resolutions and uncertainties might be achieved and what predicted seismic observable (travel-times, amplitudes, waveforms) accuracies would these imply? (Santa Barbara Room)

5:00-5:15 Closing remarks. Jay Zucca, LLNL 